\title{
EXPERIMENTAL STUDY ON CONFINED BUCKLE PROPAGATION
}

\author{
Peter Stephan ${ }^{1}$, Casey Love ${ }^{1}$, Faris Albermani ${ }^{1, *}$ and Hassan Karampour ${ }^{2}$ \\ ${ }^{1}$ School of Civil Engineering, The University of Queensland, Australia 4072 \\ ${ }^{2}$ School of Engineering, Griffith University, Australia 4222 \\ *(Corresponding author: E-mail: f.albermani@uq.edu.au)
}

Received: 9 December 2014; Revised: 7 March 2015; Accepted: 23 March 2015

\begin{abstract}
Results from hyperbaric chamber tests of confined buckle propagation using $3 \mathrm{~m}$ long aluminium pipes with three different $D / t$ ratios are presented and discussed. A new testing method, confined ring squash test, is proposed to estimate the confined buckle propagation pressure. The proposed confined ring squash test gives a reasonable estimate of the confined buckle propagation pressure and is a much expedient test to implement in comparison to hyperbaric chamber test.
\end{abstract}

Keywords: Confined buckle propagation; Hyperbaric chamber; Ring squash test

DOI: $10.18057 /$ IJASC.2016.12.1.4

\section{INTRODUCTION}

Buckle propagation is a snap through phenomenon where a local collapse once initiated may propagate rapidly along the structure with catastrophic consequences. The propagation pressure $P_{p}$ is substantially lower than the pressure required for the initiation of local collapse, the initiation pressure $P_{I}$, [1]. The propagation pressure is a vital design criterion in many engineering applications such as deep subsea pipelines [2-6], heat exchangers and penstocks in hydroelectric power plants [7]. Figure 1 shows a typical propagation response obtained from testing aluminium pipes in a hyperbaric chamber [4]. The response is depicted in terms of the applied hydrostatic pressure vs the pipe's volume change. The propagation response is characterised by the initiation and propagation pressures $\left(P_{I}\right.$ and $\left.P_{p}\right)$ and by the dog-bone deformation that propagates along the pipe as shown in Figure 1. The elastic collapse pressure, $P_{c}$, represents an upper-bound on $P_{I}$ and the pressure from a ring collapse mechanism, $P_{P M}$, gives a lower-bound on $P_{p}$ [2]. These two pressures, $P_{c}$ and $P_{P M}$, are given by

$$
\begin{aligned}
& P_{c}=\frac{2 E}{\left(1-v^{2}\right)}\left(\frac{t}{D}\right)^{3} \\
& P_{P M}=\pi \sigma_{y}\left(\frac{t}{D}\right)^{2}
\end{aligned}
$$

where $E, v$, and $\sigma_{y}$ are the elasticity modulus, Poisson's ratio and yield stress of the material, respectively, and $D$ and $t$ are the pipe's diameter and wall thickness.

Another form of buckle propagation is the confined buckle propagation which can be observed when a pipe is confined (without a mechanical connection) in a close-fit rigid medium and is subjected to hydrostatic pressure. Shell liners in wells and underground shafts and slip-on buckle 
arrestor used in subsea pipelines are some examples where confined buckle propagation may take place $[8,9]$. The confined buckle propagation response is characterised by the confined initiation and confined propagation pressures ( $P_{I c}$ and $P_{p c}$ respectively) and by the U-shape (rather than a dog-bone) deformation that propagates along the confined pipe as shown in Figure 2.

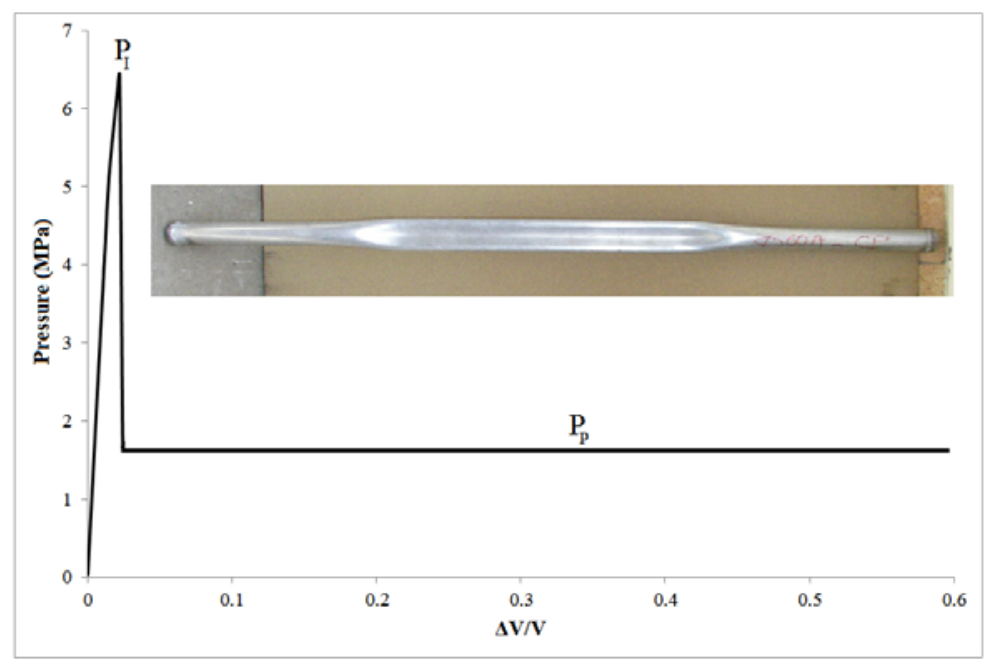

Figure 1. Buckle Propagation (Unconfined) with Dog-bone Deformation

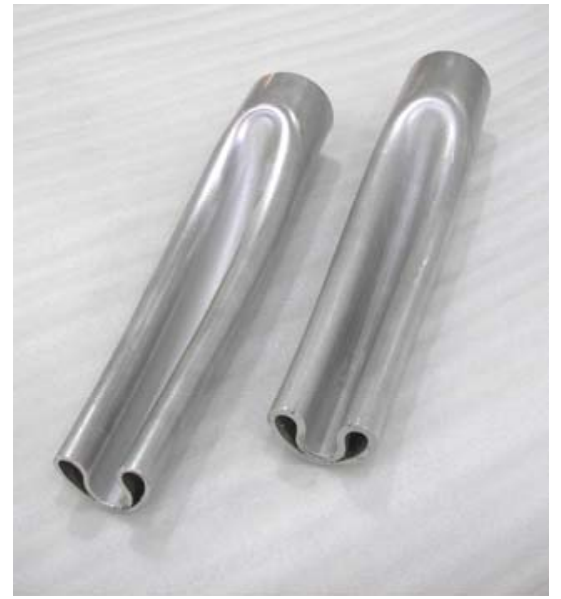

Figure 2. Confined Buckle Propagation with the U-mode Shape of Deformation

In this work, experimental investigation is conducted using $3 \mathrm{~m}$ long aluminium pipes with outer diameter of $76.2 \mathrm{~mm}$ (length-to-diameter ratio, $L / D=39.4$ ) and three different diameter-to-thickness $(D / t)$ ratios $(16.2,23.8$ and 47.6) as shown in Table 1. Three tensile coupon tests were conducted for each $D / t$ and the average obtained material properties are listed in Table 1.

Table 1. Material and Section Properties of the Test Specimens

\begin{tabular}{|l|l|l|l|l|l|l|}
\hline$D / t$ & Alloy & $\begin{array}{l}D \\
(\mathrm{~mm})\end{array}$ & $\begin{array}{l}t \\
(\mathrm{~mm})\end{array}$ & $\begin{array}{l}E \\
(\mathrm{GPa})\end{array}$ & $\begin{array}{l}E^{\prime} / E \\
(\%)\end{array}$ & $\begin{array}{l}\sigma_{y} \\
(\mathrm{MPa})\end{array}$ \\
\hline 16.2 & $6060-\mathrm{T} 591$ & 76.2 & 4.7 & 69.0 & 0.65 & 76.7 \\
\hline 23.8 & $6063-\mathrm{T} 4$ & 76.2 & 3.2 & 69.0 & 0.68 & 75.7 \\
\hline 47.6 & $6060-\mathrm{T} 5$ & 76.2 & 1.6 & 69.0 & 0.4 & 212.7 \\
\hline
\end{tabular}


A $2 \mathrm{~m}$ long seamless steel pipe with inner diameter of $77.9 \mathrm{~mm}$ and $5.5 \mathrm{~mm}$ wall-thickness is used for confining the aluminium pipe as shown in Figure 3. This gives $0.85 \mathrm{~mm}$ clearance around the aluminium pipe along the $2 \mathrm{~m}$ long confined length with the remaining $1 \mathrm{~m}$ length of the aluminium pipe is left unconfined. The flexural rigidity ratio of the confining steel pipe to the confined aluminium pipe ranges from around 5 to 118 (depending on $D / t$ of the aluminium pipe and assuming the same Poisson's ratio for steel and aluminium).

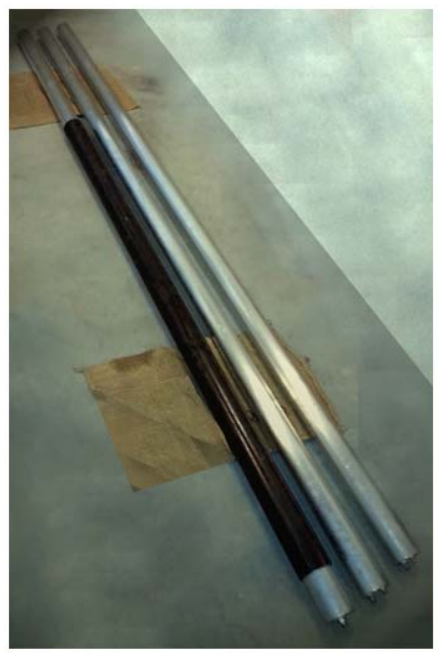

Figure 3. Some of the $3 \mathrm{~m}$ long Aluminium Pipes used in the Test, One of them is shown with $2 \mathrm{~m}$ Long confining steel pipe

\section{HYPERBARIC CHAMBER TEST OF CONFINED BUCKLE PROPAGATION}

A $4 \mathrm{~m}$ long stiff hyperbaric chamber with $173 \mathrm{~mm}$ internal diameter is used in this test as shown in Figure 4. The chamber is rated for $20 \mathrm{MPa}$ internal pressure [4]. A volume-controlled pressurization with a high pressure pump is used to obtain buckle propagation under quasi-static steady-state conditions. The $3 \mathrm{~m}$ long aluminium pipe specimen is sealed by welding a thick cap at each end. The outer wall of the aluminium pipe is lubricated and the pipe is inserted inside the $2 \mathrm{~m}$ long confining steel pipe. Two valves are connected at one end of the aluminium pipe through the stiff cap as shown in Figure 4. One valve is used for bleeding the aluminium pipe while filling it with water before testing commence. The second valve is used to vent the aluminium pipe specimen to the atmosphere through an umbilical connected through the chamber's wall. This second valve and umbilical are also used to collect water flow from the aluminium pipe specimen during buckle propagation. The amount of water flow from the aluminium pipe corresponds to volume change during buckle propagation. The aluminium pipe specimen with the confining steel pipe around it are inserted in the chamber then the aluminium pipe is filled with water and vented through the chamber wall. The chamber is sealed, filled with water then gradually pressurised.

Three control tests were conducted first (one for each $\mathrm{D} / \mathrm{t}$ ) to determine the propagation response of the aluminium pipes without the confining steel pipe. In these tests, $3 \mathrm{~m}$ long intact (as is) aluminium pipe specimens were used. The experimental propagation response obtained from these control tests is shown in Figure 5 and the obtained $P_{I}$ and $P_{p}$ are listed in Table 2 together with the lower-bound $P_{P M}$ from Eq. 2. Figure 5 shows a typical propagation response with steady increase in pressure as the chamber is gradually pressurized until the initiation pressure $P_{I}$ is reached when a section along the aluminium pipe specimen collapses leading to a significant drop in chamber's pressure and steady flow of water from within the vented pipe specimen. By maintaining a slow rate of pressurizing, the chamber's pressure is stabilized at the propagation pressure, $P_{P}$, with the 
dog-bone buckle longitudinally propagating along the pipe accompanied by steady flow of water from within the vented pipe specimen. Figure 6a shows the three pipes at the end of the test, the extent of the dog-bone buckle propagation is clearly seen in this Figure.

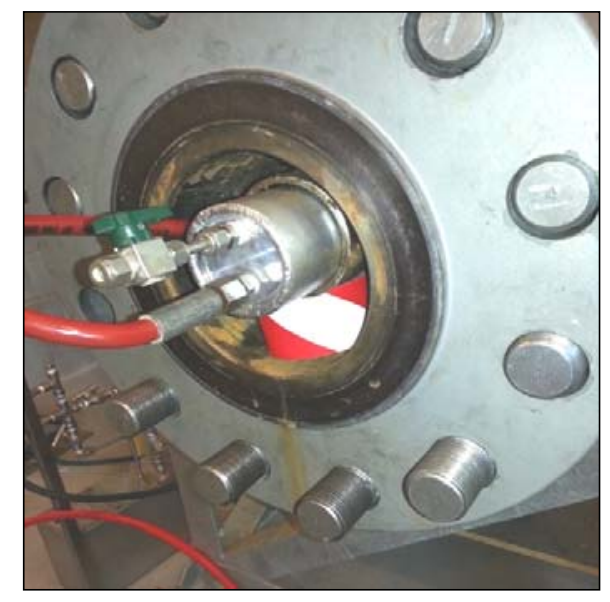

Figure 4. The Hyperbaric Chamber used in the Test

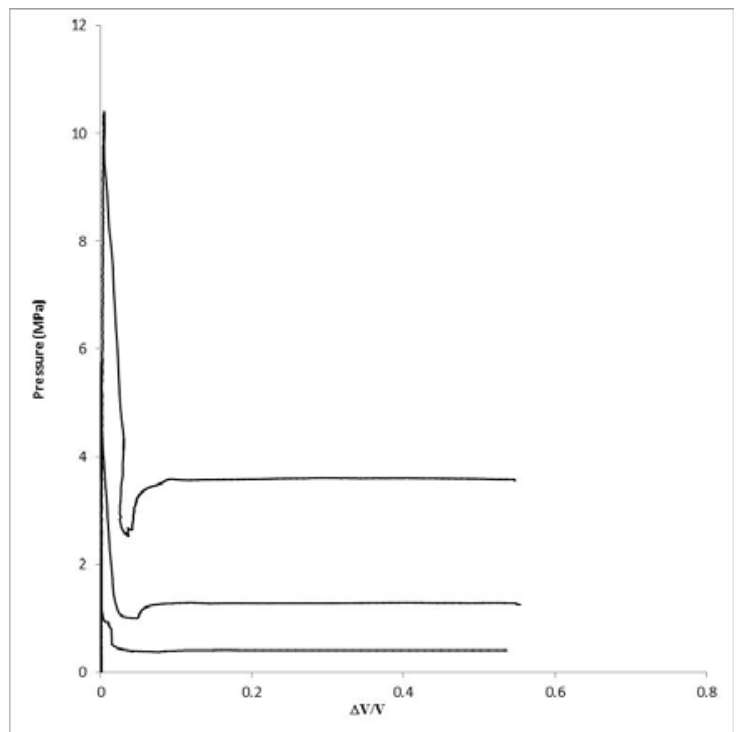

Figure 5. Buckle Propagation Response (Unconfined) from Control Tests

Table 2. Buckle Propagation (Unconfined) Test Results

\begin{tabular}{|l|l|l|l|l|}
\hline \multirow{2}{*}{$D / t$} & \multicolumn{4}{|l|}{$\begin{array}{l}\text { Buckle propagation (unconfined) } \\
(\mathrm{MPa})\end{array}$} \\
\cline { 2 - 5 } & $P_{I}$ & $P_{p}$ & $\begin{array}{l}P_{P M} \\
(\text { eq } 2)\end{array}$ & $P_{P M} / P_{p}$ \\
\hline 16.2 & 10.4 & 3.6 & 0.92 & 0.26 \\
\hline 23.8 & 5.8 & 1.3 & 0.42 & 0.32 \\
\hline 47.6 & 1.5 & 0.4 & 0.29 & 0.72 \\
\hline
\end{tabular}




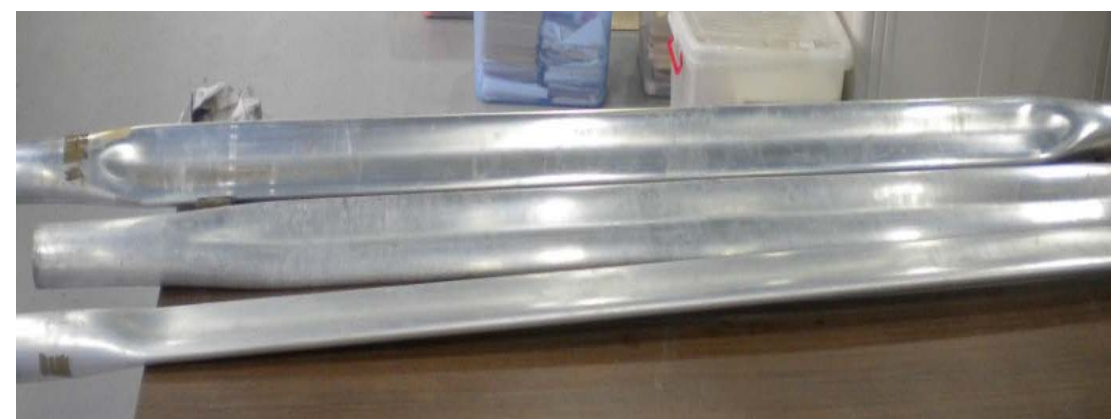

(a)

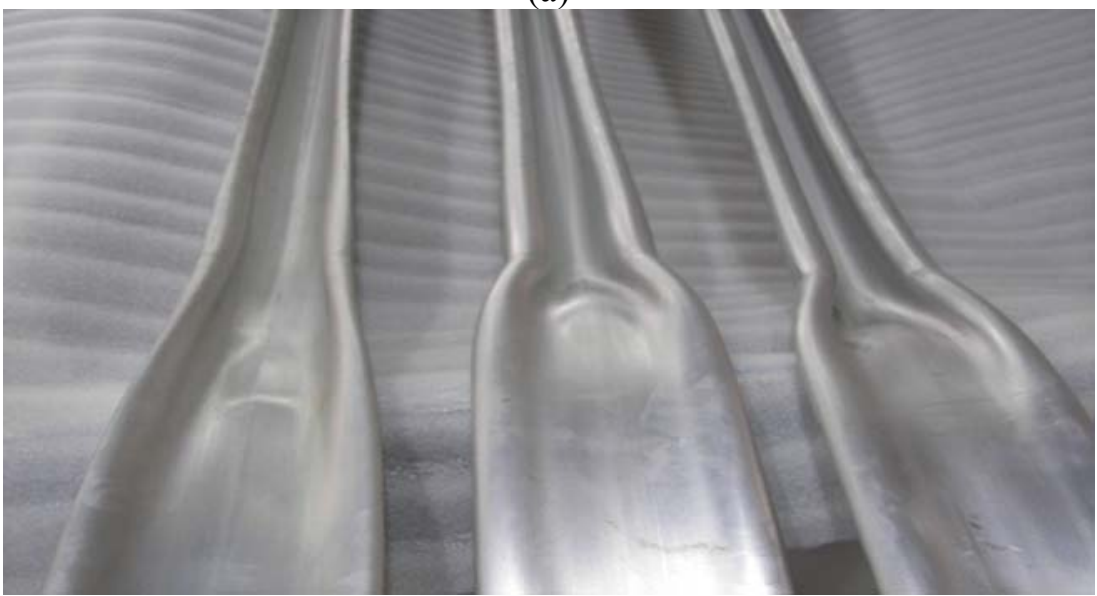

(b)

Figure 6. Deformed Specimens after Buckle Propagation; a) Unconfined, b) Confined

Similar test protocol as described above is followed for the confined buckle propagation. The $3 \mathrm{~m}$ long aluminium pipe specimen was lubricated and inserted inside the $2 \mathrm{~m}$ long confining steel pipe prior to inserting in the hyperbaric chamber. In order to insure that buckle propagation initiates at the unconfined region of the aluminium pipe, a small dent is imposed mid-way at the unconfined $1 \mathrm{~m}$ long section of the aluminium pipe specimen. The ovalization caused by the dent is expressed as an ovalization ratio $\Omega$ and maintained at around $7 \%$ for all the test specimens. The ovalization ratio is taken as:

\section{$\Omega=\frac{D_{\operatorname{man}}-D_{\min }}{D_{\max }+D_{\min }}$}

where $D_{\max }$ and $D_{\min }$ are the maximum and minimum measured outer diameter of the dented aluminium pipe.

Eight confined tests were conducted in the hyperbaric chamber, these consist of three tests for each of $\mathrm{D} / \mathrm{t}=47.6$ and 23.8 and two tests for $\mathrm{D} / \mathrm{t}=16.2$. However, two tests for $\mathrm{D} / \mathrm{t}=47.6$ sprung a leak at the weld of the stiff end cap as the buckle start penetrating the confined section. For this reason these two tests were disregarded.

The experimental confined propagation response from the remaining six tests is shown in Figure 7 and the average obtained characteristic pressures $\left(P_{I}, P_{p}, P_{I c}\right.$ and $\left.P_{p c}\right)$ are listed in Table 3 . In these tests, the buckle is initiated first $\left(P_{I}\right)$ at the unconfined region of the aluminium pipe where the dent is imposed. The dog-bone deformation propagates at $P_{p}$ along the unconfined length until it approaches the confined region. A noticeable build-up of pressure inside the chamber is observed. When the pressure inside the chamber reaches the confined initiation pressure $P_{I c}$, the buckle 
transforms from a dog-bone to a U-shape and penetrates through the confined region. The U-shaped buckle propagates along the confined aluminium pipe at the confined propagation pressure $P_{p c}$. The transition of the buckle from a dog-bone to a U-shape can be seen in Figure 6b. Due to the acute imperfection sensitivity of buckle initiation (in contrast to buckle propagation [2-4]), the $P_{I}$ shown in Table 3 for the dented specimens is less than that shown in Table 2 for the intact pipes. In order to align the curves for different $\mathrm{D} / \mathrm{t}$ ratios shown in Figure 7 , the change in volume $\Delta \mathrm{V}$ (X-axis) is normalised by the change in the specimen's volume, $\tilde{\mathrm{V}}$, at $P_{I c}$.

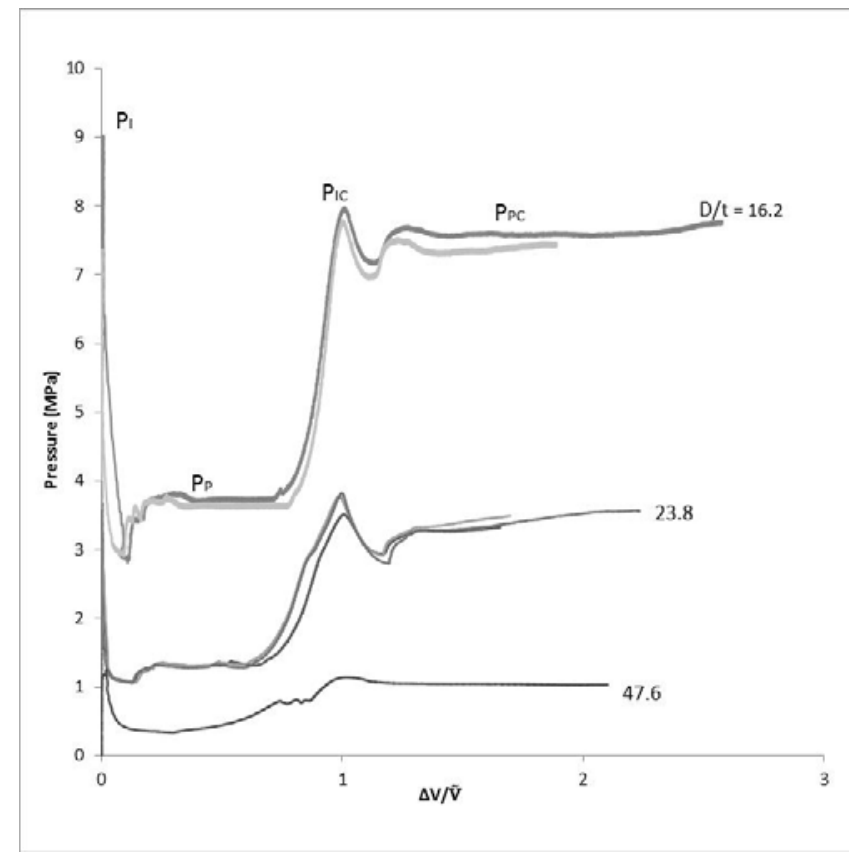

Figure 7. Confined Buckle Propagation Test Results

\section{RING COLLAPSE MECHANISM}

Assuming elastic perfectly plastic material, a ring collapse mechanism with two plastic hinges and reverse curvature of a semi-circular profile can be used to approximate the U-shaped confined propagation buckle pressure [8]. Accordingly, the confined propagation pressure from the ring collapse mechanism, $P_{p c M}$, is given by

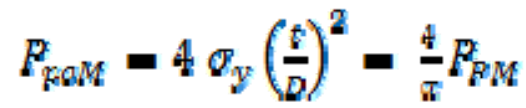

The results from eq 4 for the three $D / t$ ratios used in the hyperbaric chamber tests are listed in Table 2 , these results grossly underestimate $P_{p c}$.

The experimental results from the hyperbaric chamber tests for unconfined $P_{p}$ and confined $P_{p c}$ propagation pressures (Table 3 ) can be represented by a power law relationship [9]

$$
\frac{R}{\sigma_{y}}=A\left(\frac{t}{D}\right)^{\beta}
$$

Where $P$ above corresponds to either $P_{p}$ or $P_{p c}$. The values of A and $\beta$, respectively, from best fits of 
the hyperbaric chamber results are 124.98 and 2.815 for unconfined $P_{p}$ and 203.23 and 2.703 for confined $P_{p c}$.

The experimental propagation pressure (unconfined and confined, Tables 2 and 3 ) together with the results from Eqs. 2, 4 and 5 are shown in Figure 8. It is clear from this Figure that Eqs. 2 and 4 provide lower-bound estimate for the unconfined and confined propagation pressures, respectively. Due to the larger energy required for deformation in the confined U-shaped buckle in comparison to the unconfined dog-bone shape, $P_{p c}$ is significantly higher than $P_{p}$, particularly at higher $D / t$ range, as can be seen in Figure 8 and Table 3.

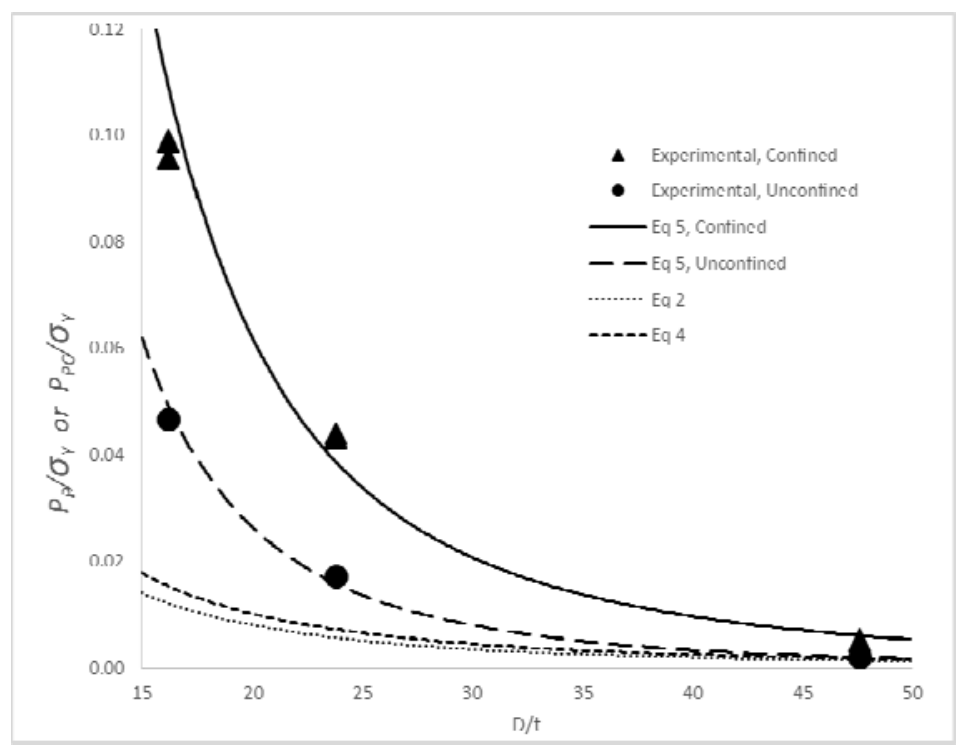

Figure 8. Summary of Experimental, Power-law Relation and Analytical Results of Buckle Propagation Pressure (Unconfined and Confined)

Table 3. Confined Buckle Propagation Test Results

\begin{tabular}{|c|c|c|c|c|c|c|c|c|c|c|}
\hline \multirow{2}{*}{$D / t$} & \multicolumn{6}{|c|}{$\begin{array}{l}\text { Confined Buckle Propagation } \\
\text { (MPa) }\end{array}$} & \multirow[b]{2}{*}{$P_{I d} / P_{I}$} & \multirow[b]{2}{*}{$P_{p d} / P_{p}$} & \multirow[b]{2}{*}{$P_{p c M} / P_{p c}$} & \multirow[b]{2}{*}{$P_{p c R} / P_{p c}$} \\
\hline & $P_{I}$ & $P_{p}$ & $P_{I c}$ & $P_{p c}$ & $\begin{array}{l}P_{p c M} \\
(e q 4)\end{array}$ & $P_{p c R}$ & & & & \\
\hline 16.2 & 8.2 & 3.6 & 7.9 & 7.5 & 1.17 & 7.09 & 0.96 & 2.08 & 0.16 & 0.95 \\
\hline 23.8 & 3.8 & 1.3 & 3.7 & 3.3 & 0.53 & 2.37 & 0.97 & 2.54 & 0.16 & 0.72 \\
\hline 47.6 & 1.3 & 0.4 & 1.2 & 1.1 & 0.38 & 0.71 & 0.92 & 2.75 & 0.35 & 0.65 \\
\hline
\end{tabular}

\section{CONFINED RING SQUASH TEST (CRST)}

The ring squash test (RST, Figure 9) has been shown to be a simple and expedient test to conduct that gives a lower bound estimate of unconfined propagation pressure $[2,4,10]$. In this test a short segment of the pipe with a length around $2-3 D$ is diametrically compressed between two semi-cylindrical rigid indenters of the same diameter and length as the pipe being tested. From the RST, and using equilibrium considerations and energy balance approach, a good estimate of propagation pressure $P_{p}$ can be calculated as described by Albermani et al [2]. 

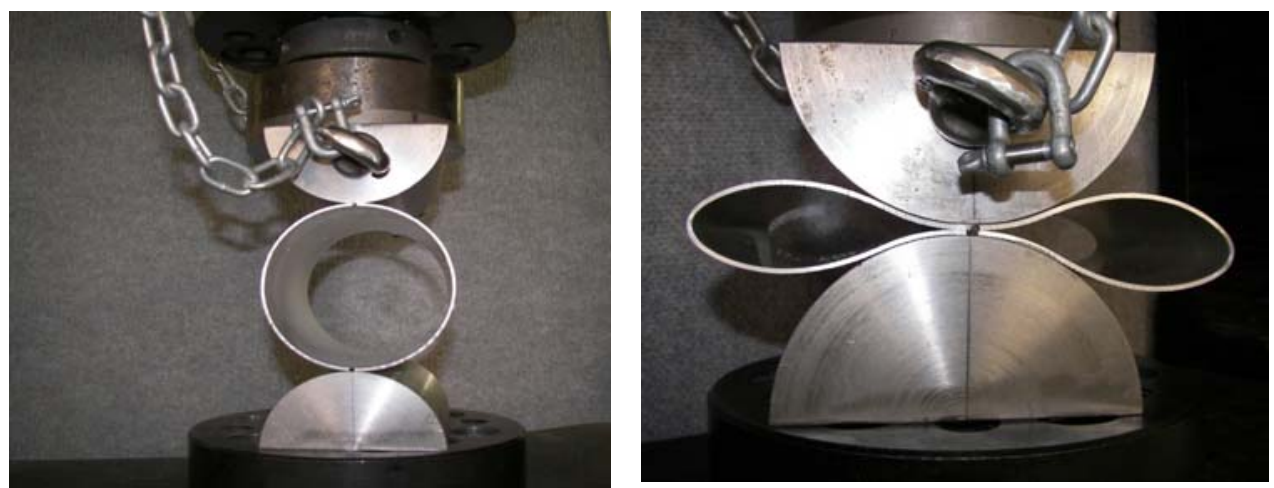

Figure 9. Ring Squash test (RST)

In this work, the RST has been modified to determine the confined propagation pressure, $P_{p c}$, as shown in Figure 10. A $2 D$ long segment of the aluminium pipe is positioned inside a semi-cylindrical solid confinement of the same diameter, $D$, as the pipe specimen and diametrically compressed by a solid semi-cylindrical indenter with a diameter of $(D-4 t)$. The length of each of the semi-cylindrical solid confinement and indenter is the same as the pipe specimen $(2 D)$.
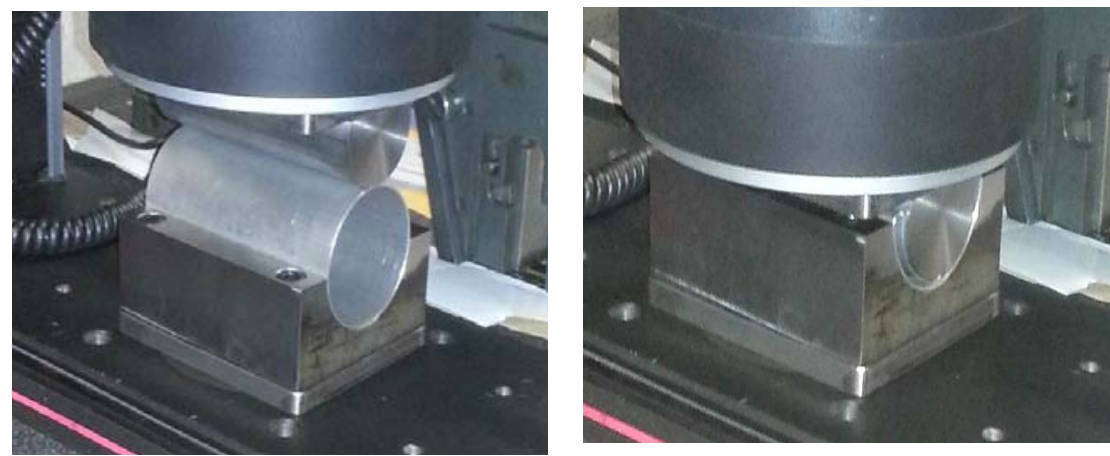

Figure 10. Confined Ring Squash Test (CRST)

Three CRST were conducted for each of the $D / t$ ratios given in Table 1 (a total of nine tests). The experimental load-displacement response from these tests is shown in Figure 11. The sharp rise in the response seen for $D / t 23.8$ and 47.6 at displacement around $70 \mathrm{~mm}$ is due to touchdown at the end of the test. In contrast, the drop in the response just before the end of the test seen for $D / t 16.2$ is due to fracture of the pipe wall at one of the fold lines of the U-shape. Figure 12 shows the nine test samples after the CRST.

By using an energy balance approach [2], an estimate of the confined propagation pressure from CRST, $P_{p c R}$, can be obtained. This is done by calculating the area, $\Lambda$, under the experimental load-displacement curve (Figure 11) and the change in the specimen's cross-section area, $\Delta A$, during the CRST (from circular to U-shape)

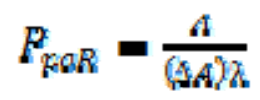

where $\lambda$ is the length of the test specimen ( $\lambda=2 D$ in the current tests). 


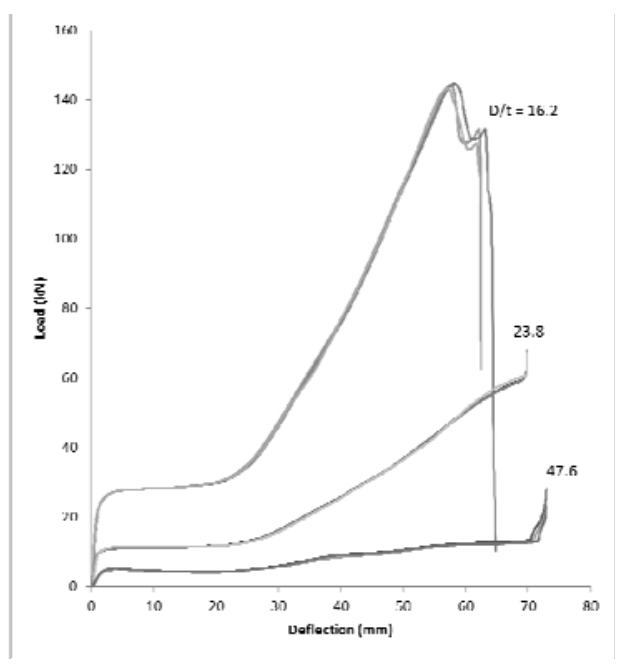

Figure 11. CRST Test Results

The average $P_{p c R}$ obtained from the CRST is listed in Table 3 (average of three tests per $D / t$ ). It is clear that the CRST provides a much better estimate of the confined propagation pressure than the ring collapse mechanism (Eq. 4).

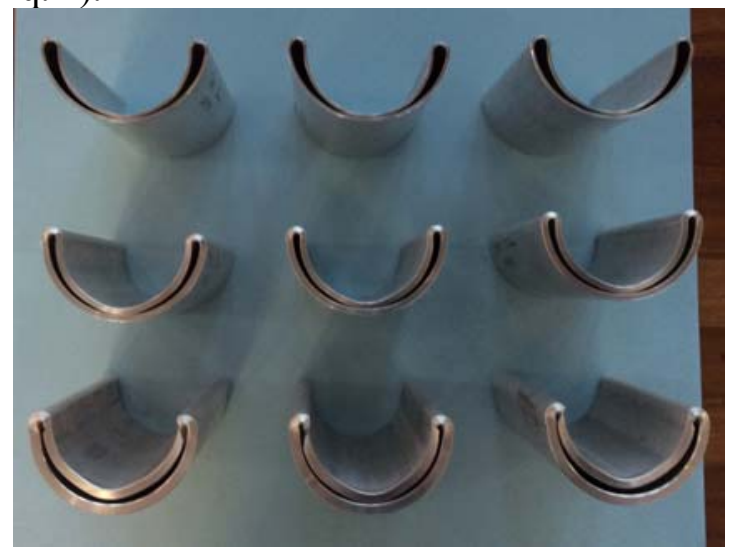

Figure 12. Test Samples (D/t: 16.2, 23.8 and 47.6) after the CRST

\section{DISCUSSION}

Hyperbaric chamber test results of confined propagation buckling show that the propagation buckle transforms from dog-bone to U-shape and penetrates through the confinement as seen in Figure $6 \mathrm{~b}$. The obtained confined initiation pressure, $P_{I c}$, is $92-97 \%$ of the unconfined initiation pressure, $P_{I}$, depending on $D / t$ ratio (Table 3 ). On the other hand, the confined propagation pressure, $P_{p c}$, is more than two times $P_{p}$. This indicates that, for the combination of $D / t$ aluminium pipes with steel confinement used in these tests, once a buckle is initiated at $P_{I}$ away from confinement, it will propagates through the confinement since $P_{I d} / P_{I}$ is less than one, regardless of $P_{p d} / P_{p}$.

As can be seen in Figures 2 and 6b, the pathological deformation experienced by the pipe as the confined U-shape buckle propagates, highlights the complex loading and mechanisms involved in confined propagation. The length, $l_{l}$, of the transition zone from dog-bone to U-shape buckle (Figure 6b) was measured for all the specimens used in the hyperbaric chamber tests. This length increases nearly linearly with $\mathrm{D} / \mathrm{t}$ as can be seen in Figure 13 with $l_{1} / D$ range from $0.5-1$. 
Another transition zone at the U-shaped wave front was also measured (Figure 2). The length of this transition zone, $l_{2}$, is taken as the distance between the crest of the collapse to the point of first contact of the opposite walls [9]. This length also increases nearly linearly with $D / t$ as can be seen in Figure 13 with $l_{2} / D$ range from 2.4-3.9. This indicates that $l_{2}$ for confined propagation buckling is shorter than that for unconfined buckling where $l_{2} / D$ is expected to be in the range of 5-11 and vary nonlinearly with $D / t[3,4,10]$. The shorter $l_{2}$ indicates that higher longitudinal stretching is involved in confined buckle propagation than in the unconfined one.

The range of $P_{p c} P_{p}$ ratio given in Table 3 is 2.08-2.75, with higher ratio at higher $D / t$. A similar trend was observed in a previous data set using stainless steel tubes [9]. The higher longitudinal stretching expected in confined propagation could be the reason for this trend since the contribution of energy dissipation by stretching in comparison to circumferential bending increases at higher $D / t$.

Unlike the ring collapse mechanism (Eq. 4), the proposed confined ring squash test, CRST, gives reasonable estimate $(65-95 \%$, Table 3$)$ of the confined propagation pressure. The CRST is a simple and expedient test to conduct in comparison to the more demanding hyperbaric chamber test.

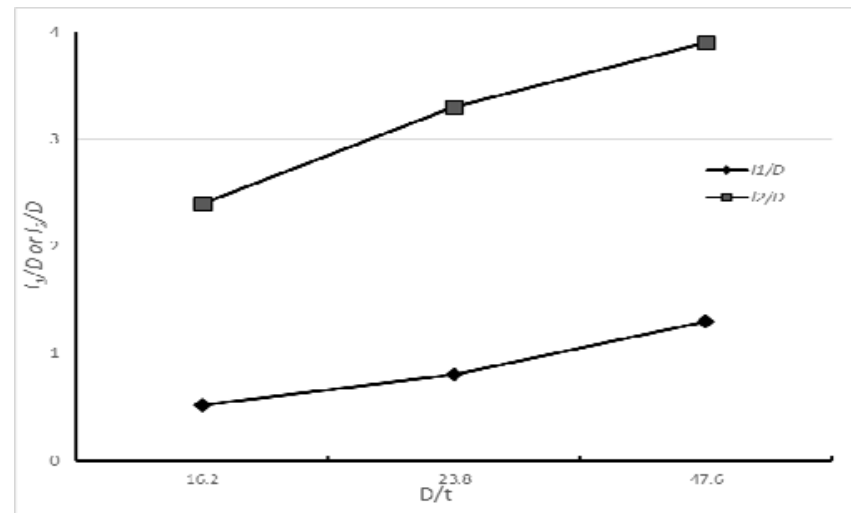

Figure 13. Transition Zone Length, $l 1$ and $l 2$, for Different $D / t$

\section{ACKNOWLEDGMENT}

We would like to acknowledge Ruth Donohoe and Christopher Russ of the Structures laboratory, School of Civil Engineering at the University of Queensland for their technical assistance in conducting the experimental work reported in this paper.

\section{REFERENCES}

[1] Kyriakides, S, "Propagation Instabilities in Structures", Advances in Applied Mechanics, 1994, Vol. 30, pp. 67-189.

[2] Albermani, F., Khalilpasha, H. and Karampour, H., "Propagation Buckling in Deep Sub-sea Pipelines", Engineering Structures, 2011, Vol. 33, No. 9, pp. 2547-53.

[3] Khalilpasha, H. and Albermani, F., “Textured Deep Subsea Pipelines”, International Journal of Mechanical Sciences, 2013, Vol. 68, pp. 224-235.

[4] Khalilpasha, H. and Albermani, F., "Hyperbaric Chamber Test of Subsea Pipelines. Thin-Walled Structures, 2013, Vol. 71, pp. 1-6.

[5] Karampour, H. and Albermani, F., "Buckle Interaction in Deep Subsea Pipelines", Thin-Walled Structures, 2013, Vol. 72, pp. 113-120. 
[6] Karampour, H. and Albermani, F., "Experimental and Numerical Investigations of Buckle Interaction in Subsea Pipelines", Engineering Structures, 2014, Vol. 66, pp. 81-88.

[7] Zaras, J., "Analysis of an Industrial Piping Installation under Buckling Propagation", Thin-Walled Structures, 2008, Vol. 46, pp. 855-59.

[8] Tam, C.K.W. and Croll, J.G.A., "An Improvement of the Propagation Buckle Performance of Subsea Pipelines", Thin-Walled Structures, 1986, Vol. 4, pp. 423-48.

[9] Kyriakides, S. and Lee, L.H., "Buckle Propagation in Confined Steel Tubes", International Journal of Mechanical Sciences, 2005, Vol. 47, pp. 603-20.

[10] Kamalarasa, S. and Calladine, C.R., "Buckle Propagation in Submarine Pipelines", International Journal of Mechanical Sciences, 1988, Vol. 30, No. 3/4, pp. 217-28. 